\title{
Design of Tourism Information System with B/S and C/S Architecture based on Android and Web Platform
}

\author{
Xuechen Dou \\ Tianjin University of Science \& Technology, Tianjin, China \\ E-mail: douxuechendmail. tust.edu.cn
}

Yonggang Yang ${ }^{\mathrm{a}}$; Weibin Zhou ${ }^{1 \mathrm{~b}}$; Zilong Zhao ${ }^{\mathrm{c}}$

Tianjin University of Science \& Technology, Tianjin, China

E-mail: ${ }^{a} 1036837358$ dqq. com; ${ }^{b}$ zhouweibinetust. edu.cn; ${ }^{c 4383006370 q 9 . c o m}$

\section{Xiaoyan Chen ${ }^{\text {; }}$ Guodong You ${ }^{\mathrm{e}}$}

Tianjin University of Science \& Technology, Tianjin, China

E-mail: ${ }^{d}$ cxywxratust.edu.cn; ${ }^{e}$ yougdongltust.edu.cn

\begin{abstract}
In order to solve the existing problems on tourism information system, a new tourism information system with $\mathrm{B} / \mathrm{S}$ and $\mathrm{C} / \mathrm{S}$ architecture is present. [Method] The system adopted $\mathrm{B} / \mathrm{S}$ and $\mathrm{C} / \mathrm{S}$ mixed architecture based on Android and web platform to ensure stable and secure operation of system. SIP protocol, RTP protocol and JNI technology were applied for system implementation. [Results] The whole system platform consists of three layers: the upper layer uses the $\mathrm{B} / \mathrm{S}$ architecture to implement the travel information Web; the middle layer realizes the sharing of the upper and lower information resources by the shared database; the lower layer adopts $\mathrm{C} / \mathrm{S}$ architecture to acquire the tourist information through the user APP based on Android platform; and $\mathrm{B} / \mathrm{S}$ and $\mathrm{C} / \mathrm{S}$ interact with each other in the mixed structure. Furthermore, the SIP protocol and RTP protocol JNI technology improved poor adaptability of the WEB interface for mobile terminal, simple expression and lack of scenic spot and traffic evaluation. [Conclusions] The present system has a lot of advantages of both $\mathrm{B} / \mathrm{S}$ and $\mathrm{C} / \mathrm{S}$ architecture and will be of great value for large-scale information systems development.
\end{abstract}

ISCC2017

16-17 December 2017

Guangzhou, China

${ }^{1}$ Corresponding author 


\section{Introduction}

With the improvement quality of people's life, tourism has gradually become a fashion. There is a large number of travel software on the market, but the majroity are the WEB pages based on the $\mathrm{B} / \mathrm{S}$ structure, which isuncomfortable for the Android mobile terminal due to poor adaptability. The expression of tourism information is text and picture, and it is simple. People need a variety of expressions, such as audio and video, the comment of Scenic spots and traffic. Through the analysis of Large-Scale Data Management System [1] and Stud-farm Daily Management System [2], the C / S architecture features such disadvantages of the narrow use, fixed user base and high maintenance costs. Based on the analysis of Book Information Retrieval System [3] and the Chain Hotel Management System [4], the disadvantages of the B / $\mathrm{S}$ architecture are the poor cross-browser experience and high costs associated with speed and security.

In order to solve the above problems, a tourism information system with $\mathrm{B} / \mathrm{S}$ and $\mathrm{C} / \mathrm{S}$ architecture based on Android and web platform was designed to solve these problems on the basis of SIP [5] protocol, RTP [6] protocol and JNI [7] technology.

The whole system platform is divided into three layers, specifically speaking, the upper layer uses the $\mathrm{B} / \mathrm{S}$ architecture to implement the travel information Web; the middle layer realizes the sharing of the upper and lower information resources by shared database and the lower layer adopts $\mathrm{C} / \mathrm{S}$ architecture to acquire the tourist information through user APP based on Android $[8,9]$ platform as the user client, and $\mathrm{B} / \mathrm{S}$ and $\mathrm{C} / \mathrm{S}$ interact with each other in the mixed structure.

\section{Key Technologies}

We present a tourism information system with $\mathrm{B} / \mathrm{S}$ and $\mathrm{C} / \mathrm{S}$ architecture based on Android and web platform and the basic structure of the $\mathrm{B} / \mathrm{S}$ and $\mathrm{C} / \mathrm{S}$ mixed communication architecture is showed in Fig.1. The upper layer $\mathrm{B} / \mathrm{S}$ architecture contains tourism information in WEB pages. The middle layer, as a shared database, contains the spot information database, scenic spot comment database and traffic comment database. Android, as an operating system platform, has good prospect and expansibility. To meet the various requirements and better experience of users, we designed a tourist information user APP based on Android system with SIP protocol, RTP protocol and JNI technology as the lower layer with good interaction interface and expansibility. And it also contains a tourism server made of such four parts as user server, register server, proxy server and redirect server with SIP protocol. Meanwhile we utilized and improved the tourism server as audio and video transmissions sever. The tourism server provides solutions for streaming file by SIP protocol and RTP protocol to provide media communication, and JNI technology to package the cross-platform open source project to be ported to Android, which can improve the speed of the application running and provide more comprehensive functions. We can use any terminal with Android operating system as the client to decode and play the audio or video and receive other instructions from the upper layer. The Android client can also communicate with the server or add new other Android clients as friends according to their own requirements. 


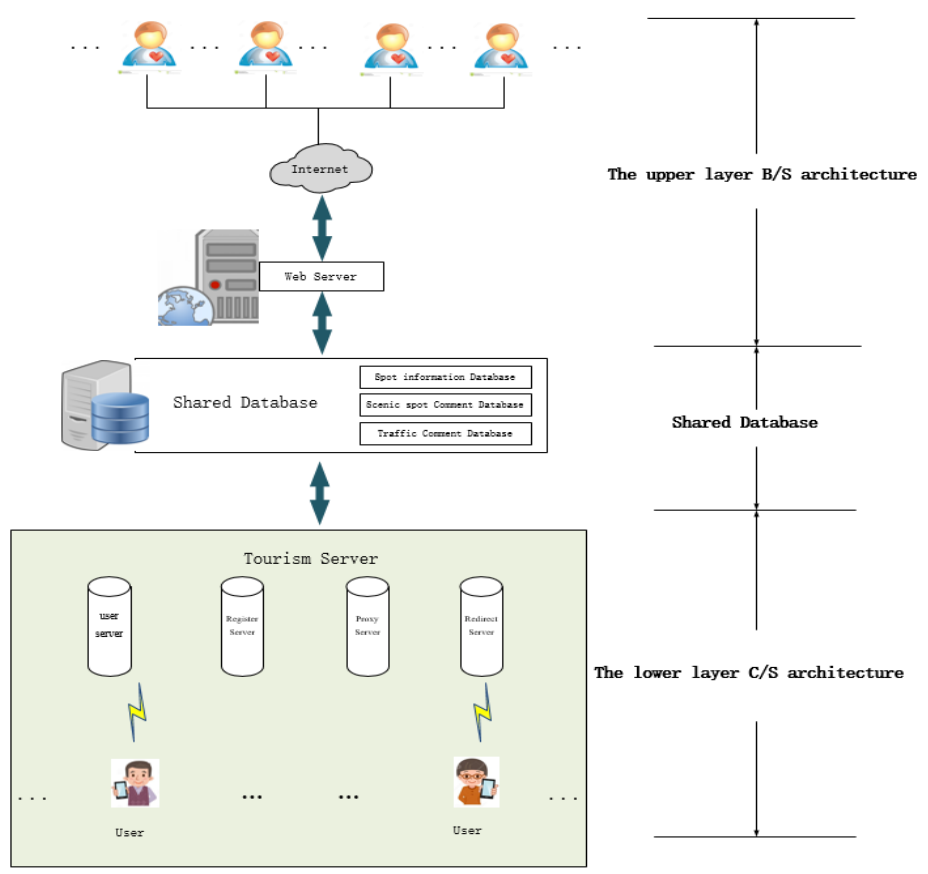

Figure 1: The $\mathrm{B} / \mathrm{S}$ and $\mathrm{C} / \mathrm{S}$ Mixed Communication Architecture

The proposed tourism information terminal containes a lot of key technologies, which include the $\mathrm{B} / \mathrm{S}$ architecture, the $\mathrm{C} / \mathrm{S}$ architecture, RTP protocol and JNI technology.

\subsection{The B/S Architecture}

The upper $\mathrm{B} / \mathrm{S}$ architecture mentioned in this paper is developed by win-form application based on $\mathrm{C \#}$ programming language in the.NET platform for the client computer system. It includes four modules: scenic spot information, scenic spot evaluation, traffic evaluation and system setting. Different modules are interacted by different SIP commands and will be required to transmit data to the underlying $\mathrm{C} / \mathrm{S}$ architecture.

\subsection{The C/S Architecture}

The lower $\mathrm{C} / \mathrm{S}$ architecture mentioned in this paper is developed by JAVA programming language on Android operating system. It contains the tourism server and the Android client, in which, the former is made of four prts including user agent, register server, proxy server and redirect server with SIP protocol. The sessions may consist of message, audio intercom, video intercom and video conference, etc. We design a $\mathrm{B} / \mathrm{S}$ and $\mathrm{C} / \mathrm{S}$ mixed communication architecture containing four servers to be synthesized in one server. The user agent contains the user log module and the user register module, the former of which is applied by the user client by user server. If the users don't register in the application and the tourism server has no data about this user, the user will register new name and password by the register server. Simultaneously, the tourism server will query the database to avoid duplicate user names. The user client communicates with proxy server and redirect server, and they make corresponding judgment on the instructions issued by the SIP signaling of user client.

We can use any Smart phone with Android operating system as the client to install the open source project application. The application includes local database, audio and video player and other features. The program supports multiple cross-platform programming languages through JNI technology. The program accesses the server to query text, pictures, comments of Scenic spots and traffic, audio, or video information about travel through the network, Play audio or video by using a player with a smart phone based on the android operating system. We 
designed a compression and decompression module with SIP protocol and RTP protocol to reduce the footprint of mobile phone memory, improve operational efficiency and enhance the fluency of audio and video playback. The user clients can use each others' user information to complete the detail sessions, such as message, audio intercom, video intercom, etc.

\subsection{RTP Protocol}

RTP [10] , the abbreviation of Real-time Transport Protocol, was proposed in 1996 by the IETF's multimedia transmission working group. The protocol provides a time stamp for synchronization, a serial number for dropping packets and reordering tests and a load format for encoding the data. Each user client sends instructions to the audio or video conversation via the SIP protocol, and after getting the permission of $\mathrm{B} / \mathrm{S}$ architecture or $\mathrm{C} / \mathrm{S}$ architecture to start media communication. The RTP protocol is used for the real-time conversation.

\subsection{JNI Technology}

$\mathrm{JNI}[11,12]$ is a programming framework that can provide interface between Java and $\mathrm{C} / \mathrm{C}+$ +. The real-time communication is based on SIP protocol and RTP protocol and it can't be implemented directly through the JAVA programming language. Therefore, we design the local $\mathrm{C}$ function as the dynamic link libraries to the *.so format. The interaction between $\mathrm{B} / \mathrm{S}$ architecture and $\mathrm{C} / \mathrm{S}$ architecture starts with JNI technology.

\section{Implementation of the Entire System}

In the preceding part, we mention that the tourism information system is divided into three parts, which are interrelated and interactive between the three parts. The APP mobile terminal and the PC client share the database and interact with each other through the network in the same LAN. The APP mobile terminal sends SIP commands to the web server and then will query database server with My SQL database. If the instruction needs to query the PC's information, it will also be sent to the PC client to achieve the goal. We describe the response flow chart of the whole system after the user sends the instruction in Figure 2.

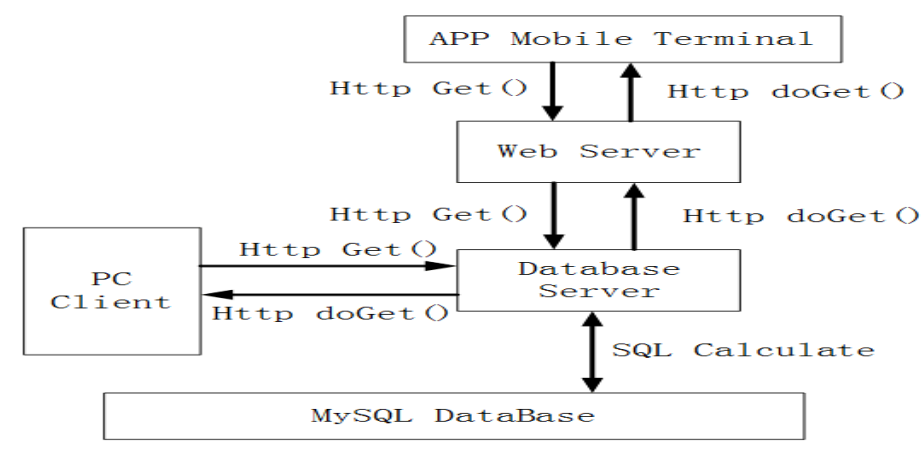

Figure 2: Response Flow Chart of the Entire System

In the whole system, SIP is primarily responsible for signaling control. The architecture is made of SIP module, RTP module, media module, local database module and other modules. The SIP module is used to achieve signaling control as the command layer. The other modules are responded to the instructions of the SIP module.

In this paper, the system superinduces audio and video information to describe scenic spots for different user groups. Moreover, we combine audio or video intercom to describe the 
progress of communication between different user clients. If the user wants to know the information of tourism spots through listening the audio, watching the video or communicating with others, the detailed work for tourism information terminal is shown as follows:

i) Through the tourism server and SIP instruction communication, the Android client acquires the permission of the server. Meanwhile, the client sends its own IP address and communication port to the server.

ii) With SIP instruction communication, the tourism server knows the client demands to have a media communication.

iii) Finally the Android clients start the communication with necessary RTP module, audio module or video module to achieve the audio or video session.

However, the tourism information terminal also supports the conversations between different inter-regional users who can share their feelings and experience with others through audio or video sessions. The detailed process of starting an audio or video session is showed in Fig. 3. If the users want to start media communication, they have to be friends first.

i) User A sends its own IP address, media port oradds a new friend requisition to the tourism server. Simultaneously the server gets user B's IP address and media port.

ii) User B confirms whether to add the new friend. The server gives feedback and sends User B's IP address and media port to user A. Meanwhile, the server makes the same instruction to User B.

iii) User A sends the audio or video command to the tourism server after tourism server has determined that the request is valid, then the request is sent to user B.

Ii-ii) The session starts between User A and User B. When the session finishes, both User A and User B send the end message to the tourism server.

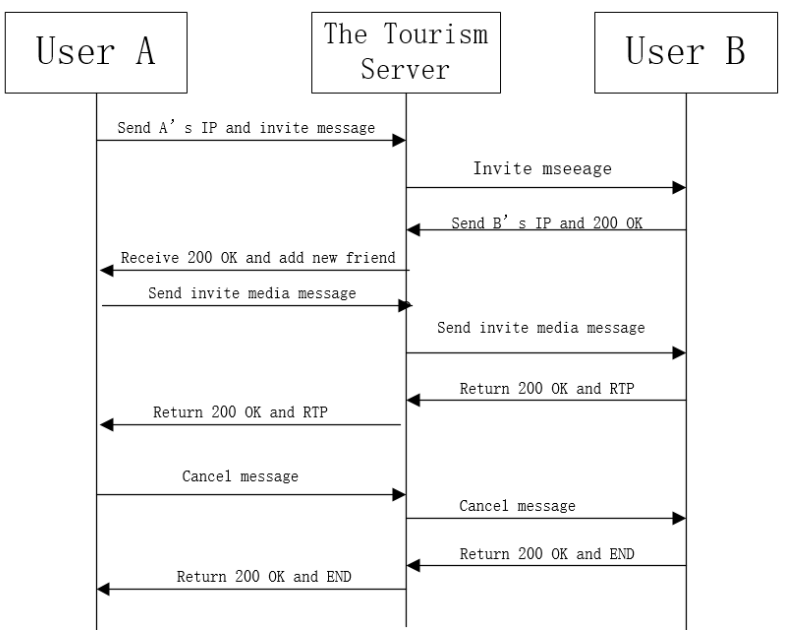

Figure 3: Process for Starting A Media Session

According to different SIP instructions sent from the underlying structure, the tourism server reacts accordingly and returns the result after quering about the upper structure. At present, the market lacks complete information display and evaluation system of scenic spots. In this paper, we also display the list of the scores of each scenic spot through the upper computer system and sort them as per the order of scores in the community and traffic page of the lower level Android software. If the tourists want to visit a scenic spot, the most important thing is the planned travel route. This system sets up traffic evaluation of each spot, thus the users can understand their own traffic information and experience through the evaluation of the corresponding spot, also pass through the functional understanding of some classical traffic to better travel routes. 
In the whole structure, Android application layer is programmed in the JAVA language and there is no module in the system that can directly make audio and video sessions. Therefore, we must write the audio and video conversation module and the comment module based on the SIP protocol and RTP protocol in C language to start the audio and video session, the scenic spot comment and the traffic comment. And then use the JNI interface to implement the audio and video session on the Android system. Figure 4 shows the implementation of the system structure.

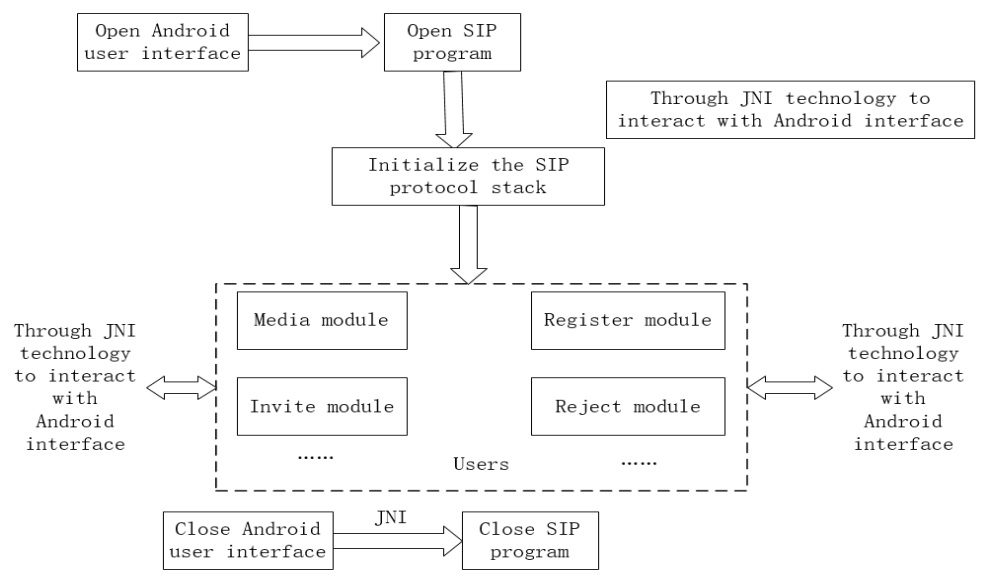

Figure 4: Response Flow Chart of the Entire System

JNI, as the bridge between audio and video of C language based on SIP protocol and the Android application layer, is also the control center of two modules. In this paper, we used JNI technology to achieve the SIP protocol and the RTP protocol. First of all, the interface section of Android application layer was defined by JNI and realized the interaction between the JNI and Android application layer. Then the audio and video conversation module based on the SIP protocol and RTP protocol in C language was realized and it can react with the SIP command. Finally, the JNI program encapsulated the Tourism.mk program as a dynamic link library into the *.so format and uses it. The file in the *.so format of JNI is equivalent to the file in the .dll format of Windows system.

\section{References}

[1]Zhan Kun Zhao. Design and Implementation of Large-Scale Data Management System Based on C/S Structure[J]. Applied Mechanics and Materials,2014,3365(602):.

[2]Luan Hong-liang, Wang Hong-bin,Qin Hong-yu,Wang Chao,Zhai Zhi-nan,Xiao Jian-hua. Design and Implementation of Stud-farm Daily Management System Based on C/S Structure[J]. Journal of Northeast Agricultural University (English edition),2014,21(3):.

[3]Shi Qang Chen,Bin Ying Wu. Design and Application of Book Information Retrieval System Based on B/S[J]. Advanced Materials Research,2013,2657(798):

[4]Nian Tao Jiao,Li Xu. Design and Implementation of the Chain Hotel Management System Based on B/S Structure $[J]$. Applied Mechanics and Materials,2014,3082(543):.

[5]Information on htto://tools.itef.org/html/rfc3261. "RFC 3261, SIP: Session Initation Protocol".

[6]Larry L.Peterson, Bruce S.Davie, Computer Networks: A Systems Approach[J], Morgan

Kaufmann Publishers Inc. , 2000 , 36 (4) :169-305.

[7]Sylvain Ratabouil. Android NDK Beginner's Guide[M]. Packt Publishing, 2012, 1, 26. 
[8]Weibin Zhou, Design of Video Indoor Phone of Android Building Intercom System [C],

proceedings of the 2012 International Conference on Frontiers of Energy and Environmental Engineering, ICFEEE 2012, p 313-315.

[9]He, Keyi, Design and implementation of SIP protocol stack based on Android[C], Proceedings 4th International Conference on Computational and Information Sciences, ICCIS 2012, p 852-855, 2012.

[10]Colin Perkins. Rtp: “Audio and Video for the Internet”.Addison-Wesley Professional, Boston, 2003.

[11]Sayed Hashimi, Pro Android, Apress, New York, 2010.

[12]Weibin Zhou, Design and Implement of Signaling Control Technology of Android Building Intercom System [J], Video Engineering,2013.15 term, p118-121(In Chinese). 